\title{
Measurement of the quadriceps femoris muscle using magnetic resonance and ultrasound imaging
}

\author{
J M Walton, N Roberts, G H Whitehouse
}

\begin{abstract}
Objectives-To define a method for measurement of the cross sectional area and volume of the quadriceps femoris muscle using magnetic resonance imaging (MRI) in conjunction with stereology, and to compare the results of measurements obtained by the MRI method with those obtained by the conventional method of static B-mode ultrasound in order to evaluate whether MRI is a reliable alternative to ultrasound.

Methods-A preliminary MRI study was undertaken on a single female volunteer in order to optimise the scanning technique and sampling design for estimating the muscle volume using the Cavalieri method. Ten healthy volunteers participated in the method comparison study. Each volunteer underwent static B-mode ultrasonography, immediately followed by MRI. The cross sectional area of the quadriceps femoris was estimated at the junction of the proximal one third and distal two thirds of the thigh, and seven systematic sections of the thigh were obtained in order to estimate muscle volume by both modalities.
\end{abstract}

Results-Seven sections through the muscle are required to achieve a coefficient of error of $4-5 \%$. There was no significant difference in the cross sectional area estimates or volume estimates when ultrasound and MRI were compared.

Conclusion-Muscle cross sectional area and volume can be measured without bias by MRI in conjunction with stereological methods and the method is a reliable alternative to static $B$-mode ultrasound for this purpose.

(Br f Sports Med 1997;31:59-64)

Department of

Medical Imaging,

University of Liverpool

J M Walton

G H Whitehouse

Magnetic Resonance and Image Analysis Research Centre, University of

Liverpool, Liverpool, United Kingdom

N Roberts

Correspondence to: J M Walton, Department of Medical Imaging, University of Liverpool, PO Box 147 Liverpool L69 3BX.

Accepted for publication 11 September 1996
Keywords: Cavalieri method; magnetic resonance imaging; muscle; stereology; ultrasound.

Measurement of the cross sectional area and volume of the human quadriceps femoris muscle is useful for monitoring the effects of immobilisation on the musculature of the lower limb resulting from trauma, disease, or aging, ${ }^{12}$ as well as assessing the response of the muscle to physiotherapy and muscle bulk building programmes in sport. ${ }^{3-5}$ Although there is a direct relationship between the cross sectional area of a muscle and its ability to generate force, ${ }^{67}$ force measurements are of limited value in evaluating muscle strength if the limb involved is painful or immobilised and evaluation of muscle strength must therefore rely on measurements of muscle size. The measurement of mid thigh circumference using a tape measure provides an indirect method of measuring muscle, but has poor reproducibility and all compartments of the thigh (namely bone, muscle, fat) are included in the measurement. ${ }^{8}$ There is a need for accurate and reproducible direct methods for muscle morphometry.

Imaging techniques are available which permit the estimation of the cross sectional area of individual muscles or muscle groups. Static B-mode ultrasound has been used in many morphometric studies of the human quadriceps muscle. ${ }^{1-5}$ The technique is reputed to be highly operator dependent, and this is likely to affect both accuracy and repeatability in muscle morphometry. ${ }^{9}$ Traditional methods for the quantification of muscle cross sectional area from ultrasound images include planimetry and the use of digitising tablets. However, these methods are time consuming and prone to operator bias. In addition, there is no optimisation of the workload required to achieve a volume estimate with known precision. In recent years, such equipment has become obsolete and there has been a need to look to other imaging modalities for quantifying skeletal muscle. Magnetic resonance imaging (MRI) is now regarded as the premier modality for the imaging of human skeletal muscle and for muscle morphometry. ${ }^{1011} \mathrm{Un}$ like computed tomography, MRI does not require the exposure of the patient to ionising radiation. ${ }^{12}$

Stereological methods permit the estimation of cross sectional area and volume to a known precision and the estimates are always unbiased. The stereological method for estimating the volume of an object is named after Bonaventura Cavalieri. ${ }^{13}$ Volume is estimated by systematically sectioning an object with parallel planes a known distance apart and the application of a mathematical formula. ${ }^{1415}$ Skeletal muscle typically has an irregular profile, particularly in the extremities. Many methods of quantification involve the use of an arbitrarily selected geometric configuration..$^{1617}$ Since stereology makes no assumption regarding the geometric configuration of an object, it is a method particularly suited to the quantification of human skeletal muscle.

The purpose of this study was to apply the Cavalieri method in conjunction with modern MRI techniques to obtain unbiased estimates of the cross sectional area and volume of the human quadriceps muscle with a known precision and to compare the MRI results with those obtained using more conventional static B-mode ultrasound imaging in conjunction with the Cavalieri method. 


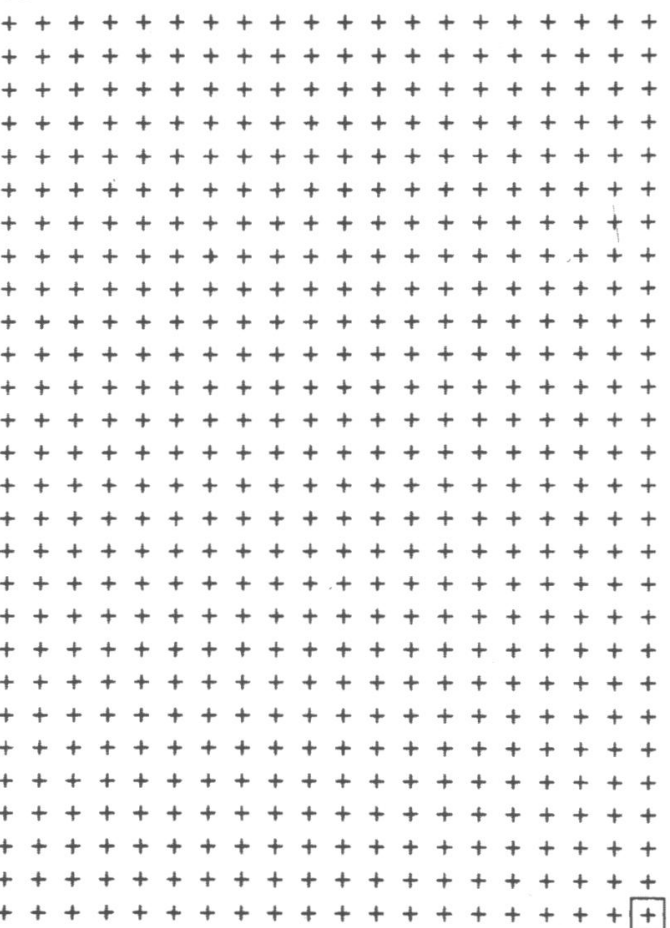

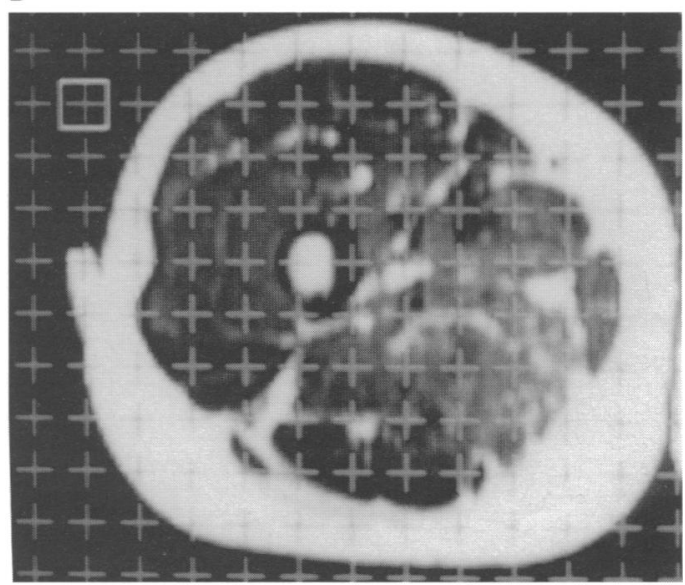

Figure 1 (A) Test system used to estimate the cross sectional area of the muscle sections on the ultrasound and MR images. The test area per test point $\left(a / p \mathrm{~cm}^{2}\right)$ used in equations 1 and 2 is the area of the small square shown in the bottom right hand corner, namely the square of the distance between the test points. In practice a similar test system was applied under computer control for the MR images and manually for the ultrasound images. (B) Test system superimposed on an image of the quadriceps muscle obtained using $M R$ (Analyze $\mathrm{T}^{\mathrm{TM}}$ software).

\section{Methods}

PRELIMINARY STUDY: OPTIMISATION OF THE SAMPLING DESIGN FOR THE UNBIASED ESTIMATION OF QUADRICEPS MUSCLE VOLUME WITH A KNOWN PRECISION BY MRI

The purpose of this study was to determine the optimum MR scanning technique for imaging the quadriceps muscle and to obtain a basic data set which could be analysed to determine the optimum sampling intensity for estimating muscle volume to a predefined level of precision using the Cavalieri method. To achieve this, more sections than would typically be required for a volume estimate were obtained.

\section{Subject, materials, and method}

A single female subject, aged 24 years, was scanned after giving informed consent. A $1.5 \mathrm{~T}$ Signa whole body imaging system (General Electric, Milwaukee, WI, USA) was employed to acquire all MR images. Imaging parameters included: fast spin echo (FSE); $T_{1}$ weighted sequence (repetition time $500 \mathrm{~ms}$, echo time $17 \mathrm{~ms}$ ) used to optimise anatomical detail; frequency bandwidth $16 \mathrm{kHz} ; 48 \mathrm{~cm}$ field of view (FOV); matrix size $256 \times 192$; number of excitations $2.0 ; 10 \mathrm{~mm}$ slice thickness; distance between sections $15 \mathrm{~mm}$; acquisition time 12 minutes.

A series of parallel axial sections of the left lower limb were obtained from the anterior superior iliac spine to the knee. Images were acquired with the subject supine and with the limb in a position of neutral abduction and slight internal rotation. The quadriceps muscle was relaxed during the acquisitions. The basic data set comprised 27 sections.

\section{Image analysis}

The stereological methods employed have been described in detail elsewhere. ${ }^{18-20} \mathrm{MR}$ images were transferred to a Sun Sparc 2 workstation (Sun Microsystems) using Ana$\mathrm{lyze}^{\mathrm{TM}}$ software (Biomedical Imaging Resources, Mayo Foundation) for image analysis.

The boundaries of the muscle group were identified on each image. A regular array of crosses (test system) (fig 1A) was superimposed upon each image, with random orientation. The number of occasions $\left(\mathbf{P}_{\dot{p}}\right)$ on which a test point lay within the boundary of the muscle was noted (fig 1B).

At each anatomical level, Matheron's formula (equation $\mathrm{Al}$ in the appendix) was used to predict the coefficient of error (CE) in the volume estimate. ${ }^{1921}$ The data were used to plot a graph (fig 2) relating the CE to the number of sections required through the quadriceps muscle to achieve a known precision. The greater the number of sections through the muscle, the lower the CE. Any method used for estimating muscle volume must be sensitive to small changes in muscle bulk. A CE suited to the estimation of human muscle volume has been reported as $5 \% .{ }^{22}$ Figure 2 demonstrates that seven sections through the quadriceps muscle are required to achieve a CE of $4-5 \%$.

To determine the contribution to error of point counting, a square grid was superimposed with random position on each section. ${ }^{19}$ The number of intersections between the grid lines and the boundary of the muscle on each section were counted. A reasonable prediction of the point counting contribution can be made using equation A2 (see the appendix). These data were used to construct a nomo- 
Figure 2 The $C E$ for a given number of sections through the quadriceps muscle. The arrow indicates that to achieve a coefficient of error of 4-5\%, which is suited to muscle assessment, seven sections through the thigh are required.

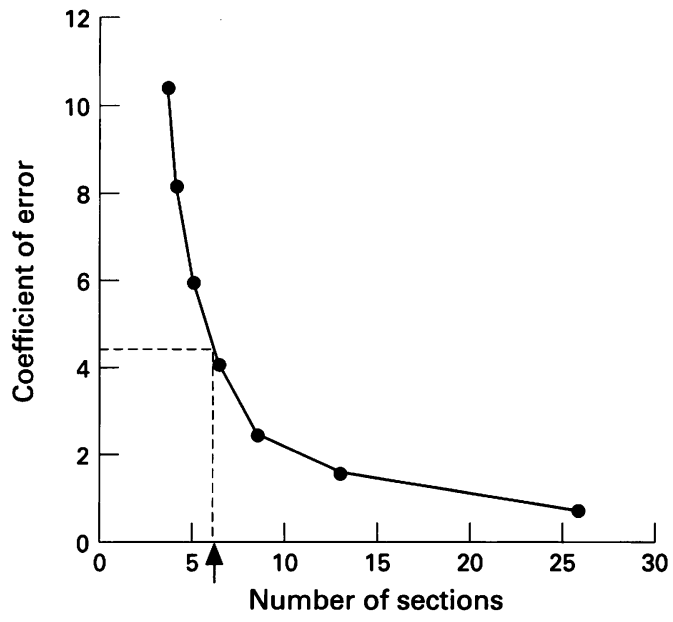

gram (fig 3), which permits a rapid prediction of the point counting contribution to the $\mathrm{CE}$ for a volume estimate of the quadriceps muscle and other muscles of similar shape. To achieve an error contribution from point counting of less than $3 \%$ for seven sections through the muscle, approximately 100 points should be counted in total for all sections through the muscle. The CE limit of 3\% has been judged to be small enough for practical purposes. If the total CE of the Cavalieri volume estimate for a muscle is for example $6 \%$, then a $3 \%$ contribution from point counting amounts to $(3 / 6)^{2}$ which equals one quarter of the total error variance, the remaining three quarters being due to section variation. ${ }^{19}$

In summary, to achieve a $4-5 \% \mathrm{CE}$ for a volume estimate of the quadriceps, seven sections

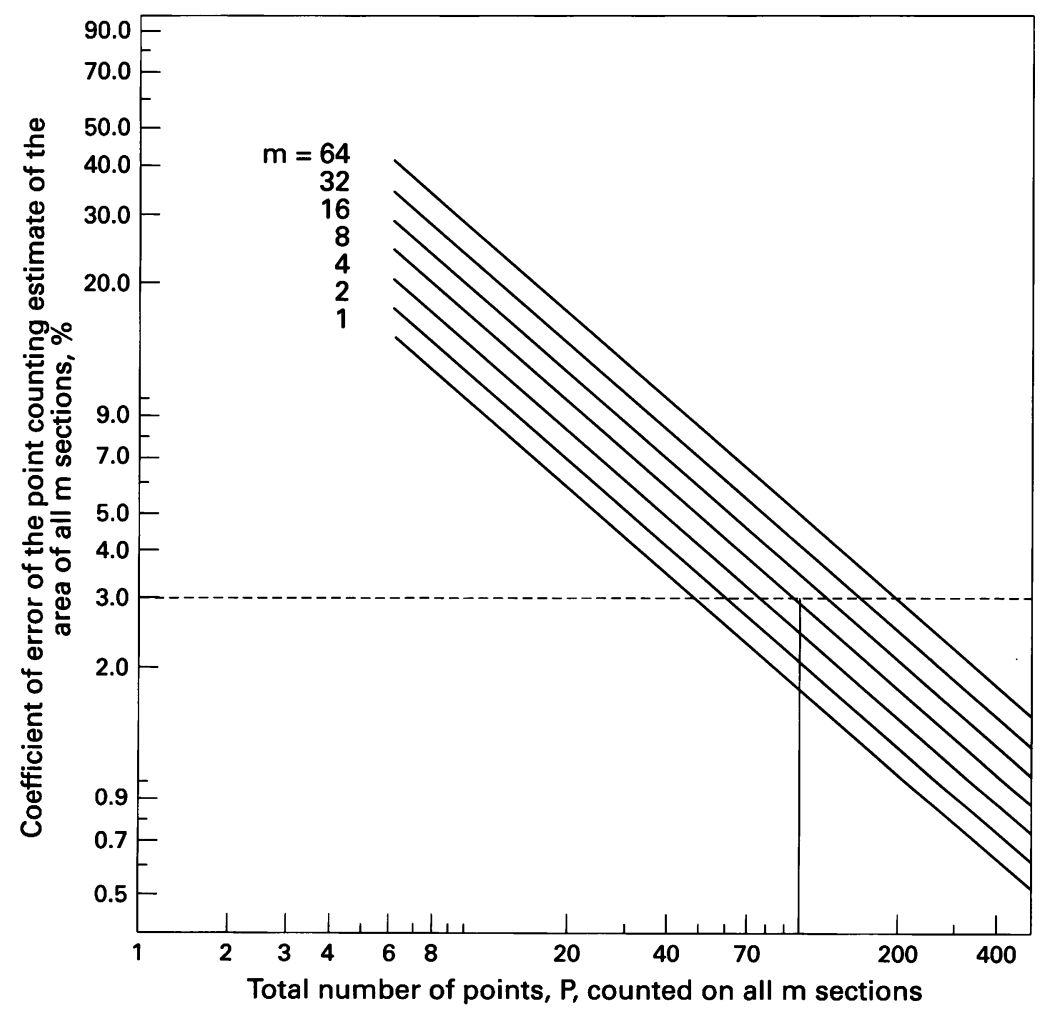

Figure 3 Nomogram for a rapid assessment of the contribution of point counting to the $C E$ for a Cavalieri estimate of the volume of the quadriceps femoris muscle. The vertical line demonstrates that approximately 100 points in total should be counted to ensure that the contribution to error from point counting is $3 \%$. through the thigh are required with 100 points being counted in total.

MAIN STUDY: COMPARISON OF ULTRASOUND AND MRI IN THE QUANTIFICATION OF THE HUMAN QUADRICEPS FEMORIS MUSCLE Subjects

Ten healthy volunteers participated in the study, after giving their written informed consent (six men, age range 19-34 years, and four women, age range 19-41 years). The left thigh was studied. There was no history of injury or pathology affecting the left thigh or lower limb and no history of neuromuscular disorders.

\section{Acquisition of ultrasound data}

Ultrasound imaging was performed immediately before MRI. All ultrasound images were acquired using a Technicare EDP 1200 static B-mode scanner (Technicare). Transducer frequency was $5 \mathrm{MHz}$ and the depth gain compensation was adjusted to optimise image quality. Hard copy images were recorded on a Sony video printer.

Each subject was scanned in the supine position with the limb fully extended, in neutral abduction and with slight internal rotation, so that the medial and lateral malleoli of the tibia and fibula respectively were equidistant from the couch. Axial images were acquired with the muscle relaxed using a direct-contact compound scanning technique. A hard copy image of each section was obtained for image analysis.

The cross sectional area of the quadriceps femoris was estimated at the junction of the proximal one third and the distal two thirds of the thigh (fig 4A).

To achieve a CE of $4-5 \%$ for the volume estimate, seven parallel axial sections through the thigh were obtained with a distance between slice centres $(T)$ of $5 \mathrm{~cm}$, and 100 points were counted in total, as determined in the preliminary study. To ensure that the volume estimate was unbiased, it was essential that the imaging sequence did not commence at the same anatomical level (for example, the greater trochanter) in each subject. The first section was therefore randomly located within the interval 0 to $\mathrm{T} \mathrm{cm}$ (fig 5).

\section{Acquisition of MRI data}

Images were acquired using the equipment and scanning sequence outlined in the preliminary study (acquisition time for seven sections was 3 minutes 19 seconds). Sections were obtained at the anatomical levels defined in the ultrasound study, with the subject in a comparable position (fig 4B).

\section{Image analysis}

The cross sectional area and volume of the muscle was estimated for both imaging modalities for each subject.

Point counting was applied to estimate the cross sectional area of the muscle at each site. The test system was applied manually for the ultrasound analysis, and a computer generated 

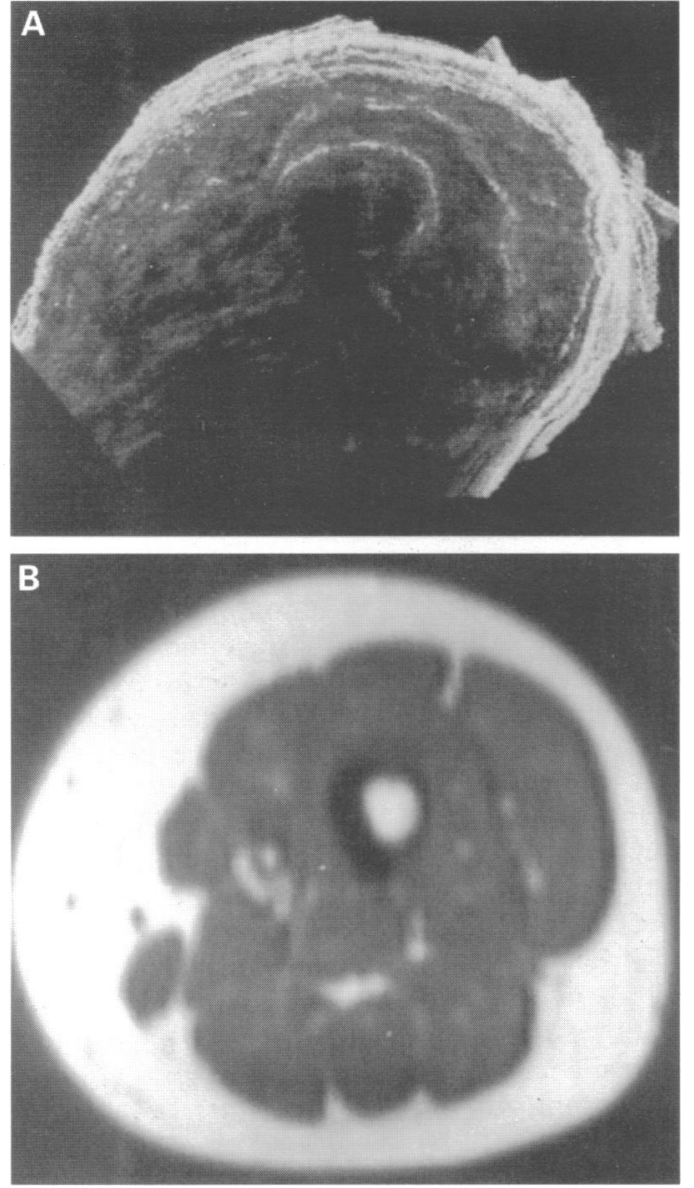

test system was generated by the Analyze program for the MRI analysis.

Estimation of muscle cross sectional area

The cross sectional area (est $A_{i}$ ) for each image was estimated using equation (1):

est $\mathrm{A}_{i}=\left(\frac{\mathrm{a}}{\mathrm{p}}\right) \mathrm{M}^{-2} \mathrm{P}_{i}$

where est $A_{i}$ is an unbiased estimate of the muscle section area $\left(\mathrm{cm}^{2}\right),(\mathrm{a} / \mathrm{p})$ is the test area per test point $\left(\mathrm{cm}^{2}\right)$ - that is, the square of the distance between the test points $\left(\mathrm{d}^{2}\right)$ (fig $1 \mathrm{~A}$ ), $M$ is the linear magnification factor for the image and $P_{i}$ is the number of occasions on which a test point falls within the defined area of the muscle boundary on each section

The values for test area per test point corrected for image magnification were $2.8 \mathrm{~cm}^{2}$

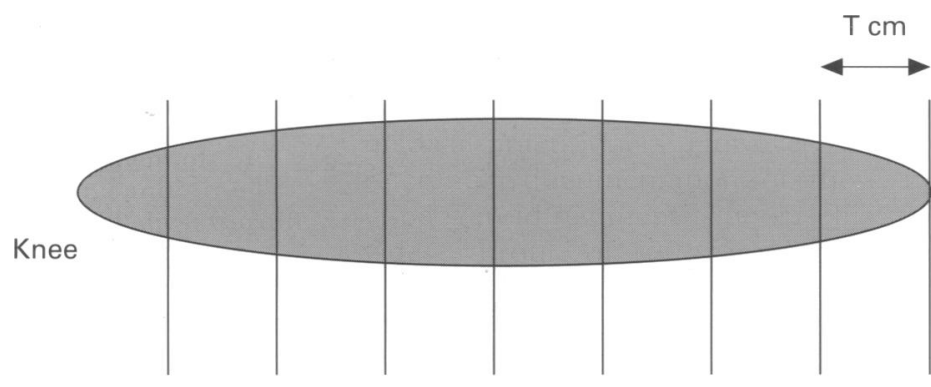

$0 \mathrm{~cm}$

Greater

trochanter

Figure 5 The Cavalieri method for volume estimation. Sections through the muscle are obtained at constant intervals (T). The first section is randomly located within the interval 0 to $T \mathrm{~cm}$. and $2.9 \mathrm{~cm}^{2}$ for the ultrasound and MRI estimates respectively.

\section{Estimation of muscle volume}

The Cavalieri method was used to estimate muscle volume (V) (fig 5). The volume of the muscle for each subject was estimated using equation (2):

est $_{2} V=T\left(\frac{a}{p}\right) M^{-2}\left(P_{1}+P_{2}+P_{3} \ldots \ldots \ldots . . . P_{m}\right)$

where est $t_{2} \mathrm{~V}$ is the unbiased estimate of volume $\left(\mathrm{cm}^{3}\right), \mathrm{T}$ is the distance between sections through the quadriceps $(5 \mathrm{~cm}),(\mathrm{a} / \mathrm{p})$ is the test area per test point $\left(\mathrm{cm}^{2}\right), M$ is the linear magnification in the image, and $P_{1}, P_{2}, P_{3} \ldots \ldots \ldots$. $P_{m}$ are the number of occasions on which a test point falls within the defined muscle boundary of the muscle in $1 \mathrm{st}, 2 \mathrm{nd}, 3 \mathrm{rd}$, and $m$ th sections.

\section{Results}

Table 1 summarises the cross sectional areas and volume estimates for both imaging modalities and each subject. A Wilcoxon matched pairs signed ranks test was applied to the data for the cross sectional area and volume estimates for ultrasound and MRI respectively. There was no significant difference in the cross sectional area $(P=0.7)$ or volume estimates ( $P$ $=0.5$ ) when ultrasound and MRI were compared (the level of significance was taken as $\mathrm{P}<0.05$ )

\section{LIMITS OF AGREEMENT}

Bland and Altman ${ }^{23}$ reported the appropriate statistical analysis for assessing the agreement between two methods of clinical measurement. This type of analysis should be undertaken to establish whether the new technique agrees sufficiently with an established technique, such that the new technique might replace the old These authors suggested that data from method comparison studies are often inappropriately analysed, notably by the use of correlation coefficients and regression analysis. The limits of agreement method of analysis has therefore been used in the analysis of the data from this study.

The paired difference between the ultrasound and MRI estimates for each subject are plotted against their mean for quadriceps cross sectional areas and volumes respectively (figs $6 \mathrm{~A}$ and $6 \mathrm{~B}$ ). The mean difference indicates the bias of the lack of agreement between the two methods. The mean difference between the ultrasound and MRI estimates of muscle cross sectional area was $-0.49 \mathrm{~cm}^{2}$. This constitutes only $0.8 \%$ of the typical cross sectional area of the quadriceps muscle $\left(60.57 \mathrm{~cm}^{2}\right)$. The mean difference between the ultrasound and MRI estimates of volume was $36.85 \mathrm{~cm}^{3}$, which constitutes $2.2 \%$ of the overall volume of a typical quadriceps muscle $\left(1659.23 \mathrm{~cm}^{3}\right)$. These differences between methods are not considered to be relevant in clinical practice. The limits of agreement $( \pm 1.96 \mathrm{SD}$ about the mean 
Table 1 Estimates of cross sectional area (CSA) and volume for the left quadriceps muscle by $M R$ and ultrasound (US) imaging

\begin{tabular}{lllll}
\hline Subject & MR CSA $\left(\mathrm{cm}^{2}\right)$ & US CSA $\left(\mathrm{cm}^{2}\right)$ & MR volume $\left(\mathrm{cm}^{3}\right)$ & US volume $\left(\mathrm{cm}^{3}\right)$ \\
\hline 1 & 72.5 & 72.8 & 1841.5 & 1876 \\
2 & 63.8 & 72.8 & 1827.0 & 1960 \\
3 & 46.4 & 47.6 & 1305.0 & 1344 \\
4 & 66.7 & 67.2 & 1943.0 & 1890 \\
5 & 69.6 & 72.8 & 1798.0 & 1778 \\
6 & 46.4 & 50.4 & 1392.0 & 1330 \\
7 & 49.3 & 47.6 & 1334.0 & 1806 \\
8 & 72.5 & 64.4 & 2146.0 & 1596 \\
9 & 58.0 & 56.0 & 1725.5 & 1372 \\
10 & 58.0 & 56.5 & 1464.5 & \\
\hline
\end{tabular}

No significant difference (Wilcoxon matched pairs signed ranks test, $P>0.05$ ) between the two methods for measurement of both cross sectional area and volume.

difference) were -9.27 and $8.39 \mathrm{~cm}^{2}$ for the cross sectional area estimates and -232.28 and $305.98 \mathrm{~cm}^{3}$ for the volume estimates.

Figure 6B demonstrates a single outlier, but despite this the limits are small enough for either the MRI method or the ultrasound method to be reliably used in clinical practice.

\section{Discussion}

The MRI technique for the unbiased quantification of the human quadriceps muscle has been defined and shown to be a reliable alternative to static B-mode ultrasound imaging for muscle morphometry. There was no
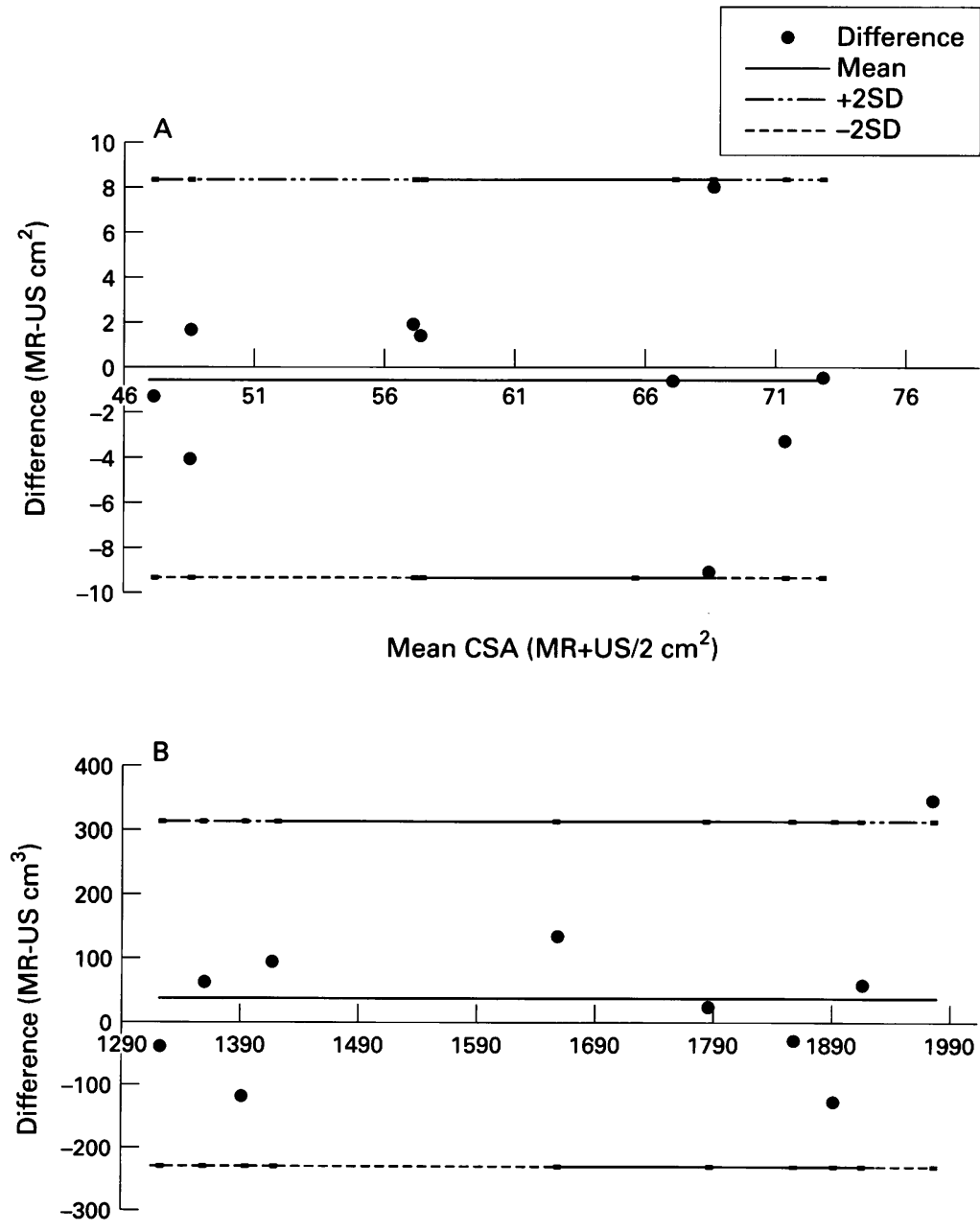

Mean volume (MR+US/2 $\mathrm{cm}^{3}$ )

Figure 6 Limits of agreement for the quadriceps cross sectional area (CSA) (A) and volume (B) measured by ultrasound (US) and MR methods. significant difference between the cross sectional area and volume estimates when the two imaging modalities were compared $(P>0.05)$.

The nomograms produced in the preliminary study (figs 2 and 3) may be referred to for optimisation of the sampling design for volume estimates of the quadriceps muscle by MRI and ultrasound, in conjunction with stereology. Since such nomograms remain constant for a given family of shapes they may also be applied to the estimation of volume for other skeletal muscles of similar shape.

MRI is recognised as the most appropriate imaging modality for muscle morphometry and has the advantage that it does not expose the subject to ionising radiation and is considered to be safe at the field strength defined in this study. ${ }^{2425}$ The soft tissue contrast is superior to that produced by any other imaging modality. ${ }^{10}$ The anatomy demonstrated on MR images of the quadriceps muscle compares favourably with that demonstrated on corresponding anatomical macroslices. The individual thigh muscles are more clearly delineated in the MR images when compared with the corresponding ultrasound images, thus permitting accurate estimation of cross sectional area and volume (figs $4 \mathrm{~A}$ and $4 \mathrm{~B}$ ). The vessels and nerves in the thigh can also be seen by MRI and excluded from the measurements. It is not possible for such structures to be clearly identified on the ultrasound images, and this may contribute to error in the ultrasound estimates. It has been suggested that MRI is the imaging modality of choice for the assessment of muscle injuries resulting from sport and for the assessment and monitoring of high performance athletes during training and rehabilitation programmes. ${ }^{2627}$ MR examinations are considered expensive and time consuming in comparison with many other imaging modalities. The development of faster scan sequences has reduced image acquisition times. MRI scanning was rapid in this experimental situation, the seven $T_{1}$ weighted images required for a volume estimate being obtained in only 3 minutes $19 \mathrm{sec}-$ onds. This acquisition time is shorter than that for the corresponding ultrasound technique, which was typically ten minutes per subject for this study. The images required for the volume estimate can be obtained in a single acquisition using multislice acquisition techniques. This minimises the scanning time required for a subject, making the technique less prohibitively expensive. However, access to MRI equipment for muscle morphometry may be restricted by the high clinical workload which is common on MRI systems.

Analysis of MR images did require the use of an expensive piece of hardware and a software package. However, images can be transferred to the workstation and analysed rapidly, with the typical image analysis time being ten minutes per muscle for a volume estimate. It would be possible to undertake volume estimation on the MR images without computer assistance, as was performed on the ultrasound images; however, this would not facilitate data processing to construct figs 2 and 3. 
Chemical shift artefacts may cause image degradation at the interface of muscle and subcutaneous fat, resulting in spatial misrepresentation of muscle boundaries on MRI ${ }^{28}$ Chemical shift artefact is worse at high field strengths but this is reduced by using higher bandwidth pulse sequences and smaller voxel sizes. This artefact was not significant with the pulse sequences and other factors used in the present study. Gradient echo sequences were avoided because chemical shift artefact can become a problem with their usage.

The Cavalieri method for volume estimates requires systematic sections through the thigh to be obtained at defined intervals. Computerised control of the MR couch permits axial sections to be obtained at defined intervals, which is difficult to achieve using static B-mode ultrasound.

The accuracy of the estimates obtained by static B-mode imaging may be influenced by extrinsic compression of the muscle by excessive transducer pressure. This is not a problem encountered with MRI as there is no direct contact with the thigh.

\section{Conclusion}

Unbiased quantification of the human quadriceps muscle can be achieved with a known precision using MRI in conjunction with stereology. It can be used to assess and monitor muscle bulk and is now a reliable and superior alternative to static B-mode ultrasound for this purpose.

We are grateful for the assistance of the radiographers from the Magnetic Resonance and Image Analysis Research Centre Professor D A Brodie for providing access to the ultrasound equipment, and Mr J R Bridson for his advice on the ultrasound study.

\section{Appendix}

Matheron's formula (equation A1) is used to determine the $\mathrm{CE}$ for the volume estimate for a variable number of sections. ${ }^{1521}$ A graph (fig 2) can then be plotted to determine the number of sections through the muscle required to estimate its volume with a known precision.

$$
\begin{aligned}
\mathrm{CE}=\left(\mathrm{est}_{1} \mathrm{~V}\right) & =\left(\sum_{i=1}^{m} \mathrm{~A}_{i}\right)^{-1}\left[\frac { 1 } { 1 2 } \left(3 \sum_{i=1}^{m} \mathrm{~A}_{i}^{2}+\right.\right. \\
+ & \left.\left.\sum_{i=1}^{m-2} \mathrm{~A}_{i} \mathrm{~A}_{i+2}-4 \sum_{i=1}^{m-1} \mathrm{~A}_{i} \mathrm{~A}_{i+1}\right)\right]^{1 / 2}
\end{aligned}
$$

where $\mathrm{CE}$ is the coefficient of error for the volume estimate and $A$ is the section area obtained from image analysis $\left(\mathrm{cm}^{2}\right)$.

The contribution of point counting to the $\mathrm{CE}$ for a volume estimate is given by:

$$
\mathrm{CE}^{2}\left(\sum_{i=1}^{m} \mathrm{p}_{i}\right)=0.0724 \frac{\overline{\mathrm{B}}}{\sqrt{\overline{\mathrm{A}}}} \times \frac{\sqrt{m}}{\left(\sum_{i=1}^{m} \mathrm{p}_{i}\right)^{3 / 2}}
$$

Where $m$ is the number of systematic sections, $P_{i}$ is the total number of points counted for all sections, $\overline{\mathbf{B}}$ is the mean bound- ary length of a section, and $\bar{A}$ is the mean section area determined by point counting. ${ }^{19}$

1 Young A, Stokes M, Round JM, Edwards RHT. The effect of high-resistance training on the strength and cross ectional area of the human quadriceps. Eur 7 Clin Invest 1983; 13:411-17.

2 Sipila S, Suominen $H$. Ultrasound imaging of the quadriceps muscle in elderly athletes and untrained men. quadriceps muscle in elderly a

3 Ikai M, Fukunaga T. A study on training effect on strength per unit cross sectional area of muscle by means of ultrasonic measurement. Internationale Zeitschrift für angewandte Physiologie einschliesslich Arbeitsphysiologie (Berlin) 1970;28 173-80.

4 Stokes M, Young A. Measurement of quadriceps crosssectional area by ultrasonography: a description of the technique and its applications in physiotherapy. Physiotherapy Practice 1986;2:31-6.

5 Rice CL, Cunningham DA, Paterson DH, Lefcoe MS. Arm and leg composition determined by computed tomography in young and elderly men. Clin Physiol 1989;9:207-20.

Schantz P, Randall-Fox E, Hutchison W, Tydén A A trand PO. O. Muscle fibre distribution, muscle cross-sectional are and maximum voluntary strength in hur

7 Narici MV, Roi GS, Landoni L, Minetti AE, Cerretelli P. Changes in force, cross sectional area and neural activation during strength training and detraining of the huma quadriceps. Eur f Appl Physiol 1989;59:310-19.

8 Nicholas JJ, Taylor FH, Buckingham RB, Ottonello D. Measurement of the circumference of the knee with ordinary tape measure. Rheumatology Disease 1976;35:282

9 Stokes M. Reliability and repeatability of methods for measuring muscle in physiotherapy. Physiotherapy Practice 1985;1:71-6.

10 Beneke R, Neuerberg J, Bohndorf K. Muscle cross-section measurement by magnetic resonance imaging. Eur $\mathcal{F} A p P l$ Physiol 1991;63:424-9.

11 Petterson H, Hamlin DJ, Manusco A, Scott KN. Magnetic resonance imaging of the musculo-skeletal system. Acta Radiol 1985;26:225-34.

12 Engstrom CM, Loeb GE, Reid JG, Forrest WJ, Avruch I Morphometry of the human thigh muscles. A comparison between anatomical sections and computer tomographic and magnetic resonance images. $\mathcal{F}$ Anat 1991;176:139-56.

13 Cavalieri B. Geometria indivisibilibus continuorum. Bononiae: Typis Clementis Ferronij, 1635. Reprinted in 1966 as Geometria degli Indivisibili. Torino: Unione TipograficoEditrice Torinese.

14 Weibel ER. Stereological methods volume 1: practical methods for biological morphometry. London: Academic Press, 1979.

15 Gundersen HJG, Jensen EB. The efficiency of systematic sampling in stereology and its prediction. $\mathcal{f}$ Microsc 1987 147:229-63.

16 Jones PRM, Pearson J. Anthropometric determination of leg fat and muscle plus bone volumes in young male and female adults. Proceedings of the Physiological Society 1969;44:63-6.

17 Weiss LW. The use of B-mode ultrasound for measuring the thickness of skeletal muscle at two upper leg sites. fournal thickness of skeletal muscle at two upper leg sites. Fournal

18 Mayhew TM, Olsen DR. Magnetic resonance imaging (MRI) and model-free estimates of brain volume determined using the Cavalieri principle. $\mathcal{f}$ Anat 1991;178:13344

19 Pache JC, Roberts N, Vovk P, Zimmerman A, Cruz-Orive LM. Vertical LM sectioning and parallel CT scanning designs for stereology: application to human lung. $f$ Microsc 1993;170:3-24.

20 Cruz-Orive LM. Systematic sampling in stereology. Bulletin of the International Statistics Institute 1993;55:451-68.

21 Matheron G. The theory of regionalized variables and its applications. Les cahiers du Centre de Morphologie Mathematique de Fontainebleau, no.5. Fontainebleau: Ecole National Superieure des Mines de Paris, 1971.

22 Roberts N Cruz-Orive LM, Reid N, Brodie DA, Bourne M, Edwards RHT. Unbiased estimation of human body M, Edwards RHT. Unbiased estimation of human body composition by the Cavalieri method using

23 Bland JM, Altman DG. Statistical methods for assessing agreement between two methods of clinical measurement. Lancet 1986;i:307-10.

24 Seidell JC, Bakker CJG, Van der Kooy K. Imaging techniques for measuring adipose-tissue distribution: a comparison between computed tomography and 1.5-T magnetic resonance. Am F Clin Nutr 1990;51:953-7.

25 National Radiological Protection Board ad hoc Advisory Group on Nuclear Magnetic Resonance Imaging. Revised guidelines on acceptable limits of exposure during magnetic resonance clinical imaging. $B r f$ Radiol 1983;56: 974-7.

26 Ehman RL, Berquist TH, McLeod RA. Imaging of the musculoskeletal system: a 5 year appraisal Radiology 1988;166:313-20.

27 Tuite MJ, DeSmet AA. MRI of selected sports injuries: muscle tears, groin pain, and osteochondritis dissecans. muscle tears, groin pain, and osteochond
Semin Ultrasound CT MR 1994;15:318-40.

28 Roberts N, Garden AS, Cruz-Orive LM, Whitehouse GH, Edwards RHT. Estimation of fetal volume by magnetic Edwards RHT. Estimation of fetal volume by magnetic resonance 